\section{RT-11 FORTRAN subroutines for on-line control}

JAMES H. HOWARD, JR

Catholic University of America, Washington, D.C. 20064

This paper outlines a package of 11 FORTRANcallable subroutines designed to enable on-line laboratory control for a wide range of psychological experiments. These routines closely parallel those reported previously for use with OS/8 BASIC on a PDP-8/e computer. They include general utilities for timing delays or displaying verbal material on a CRT terminal (Howard, 1979), as well as special-purpose routines for presenting graphic information on a Tektronix 4006 or equivalent terminal (Howard, 1981). ${ }^{1}$ The software described here, however, is coded for use with RT-11 FORTRAN IV on the popular PDP-11 laboratory computer, rather than for the older PDP-8.

Only a minimal RT-11 FORTRAN system is required. We have used both a floppy-disk-based PDP-11/03 and a larger, hard-disk-based PDP-11/23 in our laboratory. The routines use standard MACRO 11 and FORTRAN IV conventions for passing arguments (Digital Equipment

This work was supported in part by grants from the U.S. Army Research Institute and the National Institute of Mental Health to the Catholic University of America. Requests for reprints or copies of source listings should be addressed to James H. Howard, Jr., Human Performance Laboratory, The Catholic University, Washington, D.C. 20064.
Corporation, 1979), and the only supplemental hardware required is a second asynchronous interface (e.g., DLV11-J or DLV11-E) and a real-time clock (KWV11 or equivalent).

Function Summary. Table 1 presents a summary of the 11 laboratory functions with their calling syntax and arguments. Since most routines are functionally similar to those described in more detail elsewhere (Howard, 1981), only obvious differences will be considered explicitly here.

Two sets of graphic output routines are available that employ either absolute Tektronix screen addressing (MOV and DRW) or transformed screen addressing (TMOV and TDRW). The coordinate arguments for absolute addressing must be integers between 0 and 1,023. In contrast, TMOV and TDRW will accept real arguments, which will be mapped onto the absolute screen addresses automatically. The parameters of this mapping or transformation are set with the TSET routine. A call to TSET can specify an angle of rotation (ANGLE), scaling factors for the $\mathrm{X}$ and $\mathrm{Y}$ coordinates (SX and SY), and axis translation constants for both coordinates ( $\mathrm{CX}$ and $\mathrm{CY}$ ). A similar capability exists in the PDP-8 software (Howard, 1981), but the transformations are specified in a different way.

Three subroutines are provided for input from the Tektronix keyboard (TIN, NUMBIN, and SETCT). As in the $0 S / 8$ package, the TIN routine is designed to accept a single input character. The ASCII value of this character is returned as the argument NRESP with the response latency in NTIME. Although single-character

Table 1

Calling Syntax and Arguments for Each Laboratory Subroutine

\begin{tabular}{|c|c|}
\hline Syntax & Arguments \\
\hline 1. CALL DEL(N) & Delay for $\mathrm{N}$ msec \\
\hline 2. CALL CLRO & Erase Tektronix screen, no arguments \\
\hline 3. CALL ALP(N,'XXX') & $\begin{array}{l}\text { Enter alphanumeric mode and display literal character string } \\
\text { 'XXX' of length } N\end{array}$ \\
\hline 4. CALL MOV(IX,IY) & $\begin{array}{l}\text { Draw an "invisible" vector from present cursor location to } \\
\text { absolute coordinates IX, IY }\end{array}$ \\
\hline 5. CALL DRW(IX,IY) & $\begin{array}{l}\text { Draw a vector from present location to absolute coordinates } \\
\text { IX, IY }\end{array}$ \\
\hline 6. CALL TMOV(X,Y) & $\begin{array}{l}\text { Draw an "invisible" vector from present location to trans- } \\
\text { formed coordinates } \mathrm{X}, \mathrm{Y}\end{array}$ \\
\hline 7. CALL TDRW(X,Y) & $\begin{array}{l}\text { Draw a vector from present location to transformed coordi- } \\
\text { nates } X, Y\end{array}$ \\
\hline 8. CALL TSET(ANGLE,SX,SY,CX,CY) & $\begin{array}{l}\text { Set coordinate transformation parameters where: ANGLE is } \\
\text { rotation angle in radians, } S X \text { and } S Y \text { are } X \text { - and } Y \text {-coordinate } \\
\text { scaling factors, respectively, and } C X \text { and } C Y \text { specify } X \text { - and } \\
\text { Y-axis translation constants }\end{array}$ \\
\hline 9. CALL TIN(NRESP,NTIME) & $\begin{array}{l}\text { Read single input character, NRESP, record response latency } \\
\text { in NTIME }\end{array}$ \\
\hline 10. CALL NUMBIN(RESP,TIMEF,TIMEL,ITAR) & $\begin{array}{l}\text { Read multidigit number from keyboard. Numerical response } \\
\text { returned in RESP, TIMEF is latency to first digit, TIMEL is } \\
\text { latency to the last digit input, and ITAR specifies the ASCII } \\
\text { code for "enter" character }\end{array}$ \\
\hline 11. CALL SETCT(ITABLE) & Set input character translation tabie to ITABLE \\
\hline
\end{tabular}


responses are frequently required in psychological research (e.g., in a choice reaction time study), multidigit numerical responses are also common (e.g., as in a magnitude estimation experiment). The NUMBIN routine is designed to accept multidigit numerical responses. The numerical value of the response is returned as a real number in RESP, the latency to the first input digit in TIMEF and the latency to the last input digit in TIMEL. The ITAR parameter specifies the ASCII value of a terminal or "enter" character. NUMBIN will read input digits from the keyboard (nondigits will be ignored) until the "enter" character is struck.

The remaining keyboard input routine, SETCT, allows the user to "redefine" the input keyboard by assigning a new numerical value to each key. This is accomplished by passing the address of a translation table, ITABLE (an INTEGER*1 array of length 128), to the SETCT routine. Responses made after this call will produce translated values rather than their standard ASCII values. For example, the "1" key (ASCII 49)

Table 2

FORTRAN Program to Control One-Trial Magnitude Estimation Experiment

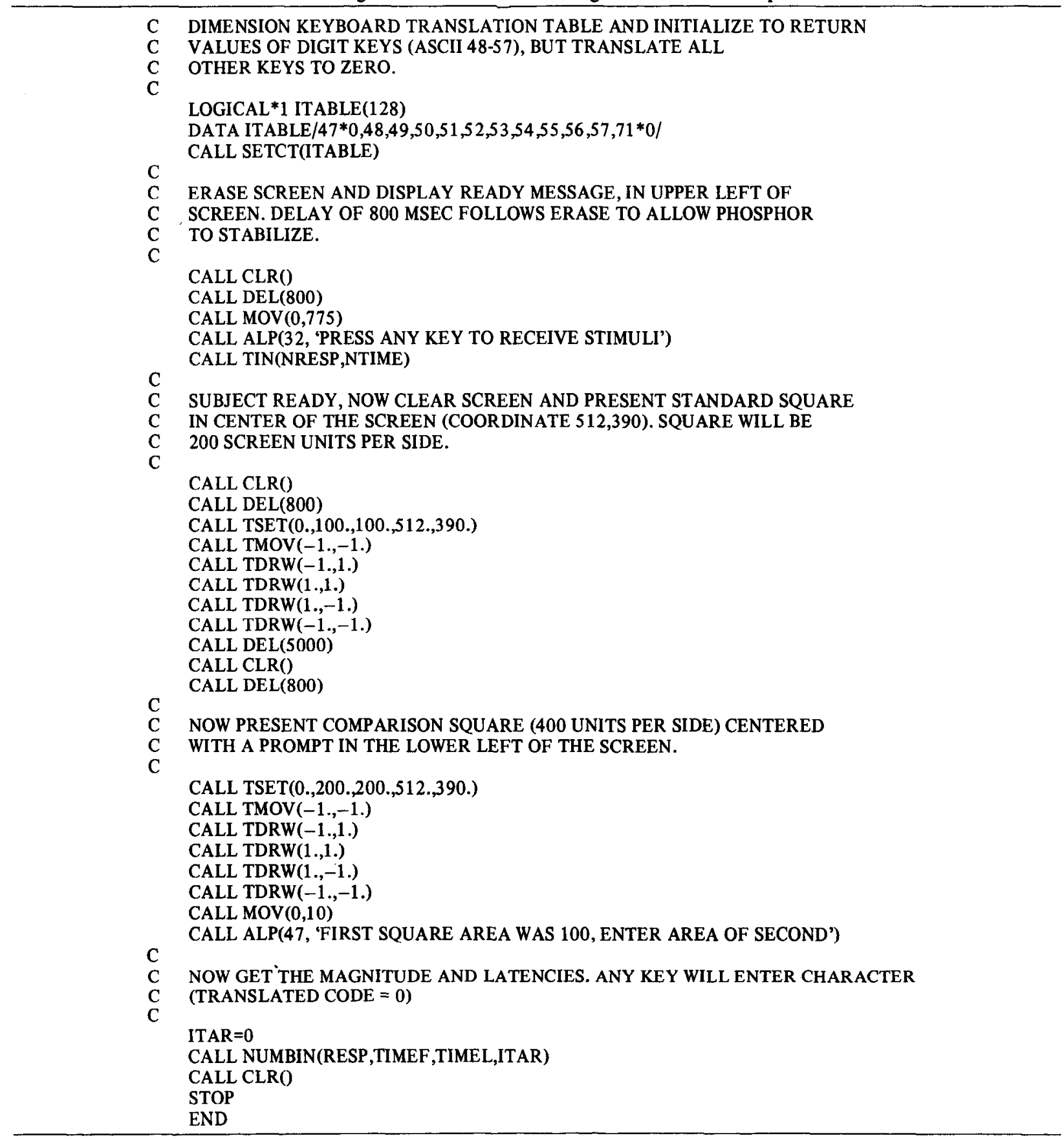


could be given a numerical value of 1 by setting the 49 th entry in ITABLE to 1 . We have found this subroutine useful for establishing a numerical keypad in the center of the Tektronix keyboard. The use of such a keypad avoids any artifact associated with the linear ordering of the digit keys in their standard locations. The Tektronix keyboard may be relabeled easily by swapping the keys.

The program shown in Table 2 illustrates how the present functions may be used for experimental control. The program controls a single-trial magnitude estimation "experiment" in which the subject is presented with two squares of different areas, a standard and a comparison. The first is displayed for $5 \mathrm{sec}$ and erased, and the second is presented. The presentation is initiated when the subject presses any key, and the magnitude estimate is entered after the second, comparison, square appears. Ten of the 11 functions listed in Table 1 are illustrated in this program.

\section{REFERENCES}

Howard, J. H., JR. Controlling the Tektronix 4006 graphics terminal with OS/8 BASIC. Behavior Research Methods \& Instrumentation, 1981, 13, 341-346.

Digital Equipment Corporation. PDP-ll FORTRAN language reference manual. Maynard, Mass: Author, 1979. How ARD, J. H., JR. A multipurpose laboratory package for OS/8 BASIC. Behavior Research Methods \& Instrumentation, 1979, $11,437-444$.

\section{NOTE}

1. The Tektronix 4000 series (4006 and 4010) terminals use storage tube graphics technology and may be limited for many applications. Alphanumeric data presentation is restricted to uppercase characters presented and displayed at a 4,800-baud rate. Since a conventional storage CRT is used, screen erasure involves energizing the entire screen. This produces a relatively bright flash, which can have an unwanted masking effect and which leaves the phosphor unstable for approximately $800 \mathrm{msec}$. These limitations are avoided with the similarly priced (approximately $\$ 3,200$ ) raster-scan CRT terminal distributed by Digital Engineering Corporation. This terminal has full upper- and lowercase alphanumeric capability, high-resolution graphics (640 by 480 ), 19,200-baud presentation rates, and nonmasking partial or whole screen erase. Although the Digital Engineering Corporation terminal is actually a modified Digital Equipment Corporation VT-100, it responds to the Tektronix 4000 series graphics protocols and may be used with the present software package. If only alphanumeric output is required, the present software may be used with any low-cost standard CRT terminal (e.g., the Zenith H-19).

(Accepted for publication April 18, 1981.) 